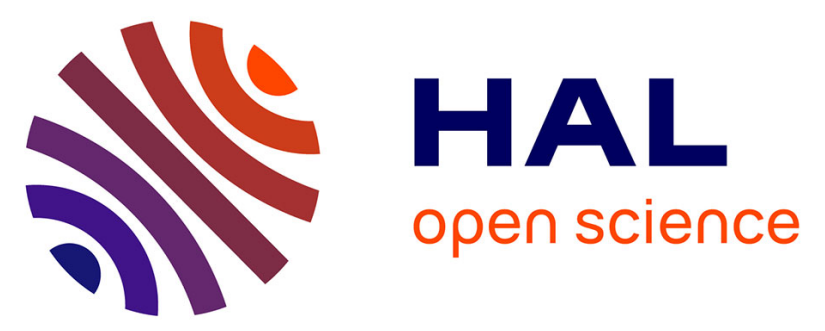

\title{
Novel Alkali Activation of Titanium Substrates To Grow Thick and Covalently Bound PMMA Layers
}

Melania Reggente, Patrick Masson, Camille Dollinger, Heinz Palkowski, Spyridon Zafeiratos, Leandro Jacomine, Daniele Passeri, Marco Rossi, Nihal Engin Vrana, Geneviève Pourroy, et al.

\section{To cite this version:}

Melania Reggente, Patrick Masson, Camille Dollinger, Heinz Palkowski, Spyridon Zafeiratos, et al.. Novel Alkali Activation of Titanium Substrates To Grow Thick and Covalently Bound PMMA Layers. ACS Applied Materials \& Interfaces, 2018, 10 (6), pp.5967-5977. 10.1021/acsami.7b17008 . hal03543591

\section{HAL Id: hal-03543591 https://hal.science/hal-03543591}

Submitted on 26 Jan 2022

HAL is a multi-disciplinary open access archive for the deposit and dissemination of scientific research documents, whether they are published or not. The documents may come from teaching and research institutions in France or abroad, or from public or private research centers.
L'archive ouverte pluridisciplinaire HAL, est destinée au dépôt et à la diffusion de documents scientifiques de niveau recherche, publiés ou non, émanant des établissements d'enseignement et de recherche français ou étrangers, des laboratoires publics ou privés. 


\title{
Novel Alkali Activation of Titanium Substrates To Grow Thick and Covalently Bound PMMA Layers
}

\author{
Melania Reggente, $\uparrow, \ddagger$ Patrick Masson, $\uparrow$ Camille Dollinger, $\S$ Heinz Palkowski, $\|$ Spyridon Zafeiratos, $\perp$ \\ Leandro Jacomine,\# Daniele Passeri, *,$\mp$ Marco Rossi, $¥$, Nihal Engin Vrana, $§, ০, \square$ Genevieve Pourroy*, $\dagger$ \\ and Adele Carrado $\uparrow$
}

$\dagger$ Institut de Physique et Chimie des Materiaux de Strasbourg (IPCMS), UMR 7504 CNRS, Université de Strasbourg, 23 rue du Loess BP 43, 67034 Strasbourg, France

Department of Basic and Applied Sciences for Engineering (BASE), Sapienza University of Rome, Via Antonio Scarpa 16, 00161 Rome, Italy

§Protip Medical, 8 Place de l'Hôpital, 67000 Strasbourg, France

„Clausthal University of Technology (TUC), IMET Institute of Metallurgy, Robert-Koch-Strasse 42, 38678

Clausthal-Zellerfeld, Germany

$\perp$ Institut de Chimie et Procédés pour l'Énergie, l'Environnement et la Santé (ICPEES), CNRS, Université de

Strasbourg, 25 rue Becquerel, 67087 Strasbourg, France

\#Institut Charles Sadron, CNRS, UPR 22, 23 rue du Loess BP 84047, 67034 Strasbourg, France

ॠCenter for Nanotechnology for Engineering (CNIS), Sapienza University of Rome, P. le A. Moro 5, 00185

Rome, Italy

-Institut National de la Santé et de la Recherche Médicale, INSERM Unité, 1121, 11 rue Humann, 67085

Strasbourg Cedex, France

¿Université de Strasbourg, Faculté de Chirurgie Dentaire, Féderation de Médecine Translationnelle de

Strasbourg, Féderation de Recherche Matériaux et Nanosciences Grand Est (FRMNGE), P. le A. Moro 5, 67000

Strasbourg, France

ABSTRACT: Titanium (Ti) is the most widely used metal in biomedical applications because of its biocompatibility; however, the significant difference in the mechanical properties between $\mathrm{Ti}$ and the surrounding tissues results in stress shielding which is detrimental for load-bearing tissues. In the current study, to attenuate the stress shielding effect, a new processing route was developed. It aimed at growing thick poly(methyl methacrylate) (PMMA) layers grafted on Ti substrates to incorporate a polymer component on Ti implants. However, the currently available methods do not allow the development of thick polymeric layers, reducing significantly their potential uses. The proposed route consists of an alkali activation of Ti substrates followed by a surface-initiated atom transfer radical polymerization using a phosphonic acid derivative as a coupling agent and a polymerization initiator and malononitrile as a polymerization activator. The average thickness of the grown PMMA layers is approximately $1.9 \mu \mathrm{m}$. The Ti activation-performed in a $\mathrm{NaOH}$ solution-leads to a porous sodium titanate interlayer with a hierarchical structure and an open microporosity. It promotes the covalent grafting reaction because of high hydroxyl groups' content and enables establishing a further mechanical interlocking between the growing PMMA layer and the Ti substrate. As a result, the produced graduated structure possesses high Ti/PMMA adhesion strength ( $260 \mathrm{MPa})$. Moreover, the PMMA layer is (i) thicker compared to those obtained with the previously reported techniques $(\sim 1.9 \mu \mathrm{m})$, (ii) stable in a simulated body fluid solution, and (iii) biocompatible. This strategy opens new opportunities toward hybrid prosthesis with adjustable mechanical properties with respect to host bone properties for personalized medicines.

KEYWORDS: PMMA-coated titanium, SI-ATRP, polymer-metal interface, adhesion, biocompatibility

\section{INTRODUCTION}

The surface modification of biomaterials by coating them with organic layers is widely studied because it confers some specific interfacial properties1,2 such as antibacterial3-6 and protein repellent7 or it improves the osseointegration between the implant and the surrounding bone.1,2,8 Moreover, the integration of implants is mostly limited by the onset of the stress shielding phenomena at the implant/bone interface, caused by a large discrepancy between their mechanical properties and densities.9 For this reason, there is a strong interest in developing composite structures with mechanical properties closer to that of the surrounding tissues.

Currently, attention is mostly focused on thin layers of molecules or polymer brushes linked on Ti1,10-12-whose applications in the biomedical field are in constant expansion13,14-and on stainless steel substrates.15,16 In this context, methodologies ensuring a strong covalent bonding between a thick polymer layer and Ti, controlling the 
interface between the metal and the polymer both in terms of strength and biocompatibility, are required. This would enable the production of sandwich structures that can control the mechanical properties, such as Young's modulus, for better physical compatibility between the implant and the host bone tissue.

In general, metal-polymer adhesion involves many types of interactions, ranging from weak interactions to covalent bonds. The first ones include the electrostatic and the hydrophobic interactions as well as the hydrogen bonds, and they are mainly due to physical adsorptions of the polymer chains onto the metal substrates.17,18 Even though these techniques allow simple fabrication of polymer-coated surfaces, their durability is strictly related to the nature of the polymer-metal interface, which strongly depends on the adhesion strength between the two components. Indeed, because chemical bonding between the metal and the polymer layer does not occur spontaneously, layered hybrid organic-inorganic materials may undergo premature and undesirable delamination phenomena, caused by the weak adhesion existing between the polymer layer and the metal,19 affecting the lifetime of the entire system.

Addressing this point, to improve the long-term stability of these hybrid structures, tethered polymers have been recently proposed as adhesive layers, inserted between the metal substrate and the polymer coating, to strengthen their adhesion.12,15,20,21 In particular, many efforts are devoted to directly synthetized polymer chains covalently grafted on metal substrates using a "grafting-from" approach. It involves the growth of polymer chains starting from initiator-modified surfaces using a suitable surface-initiated polymerization 22 and, with respect to the "grafting-to" approach,23 it promotes higher graft densities, allowing the fabrication of a denser, thicker (in the range of 50-125 nm), and more stable organic layer.24-27

Depending on the metal surface, the most widely used anchoring groups are the silanes, 6 the phosphonates, 28,29 or the thiols.30,31 Among them, the phosphonates, which have the advantage to be biocompatible and already successfully used for magnetic iron oxide contrast agent,32 appear to be very promising because they allow developing stable covalent monodentate, bidentate, and tridentate bonds.33,34

In this context, here we report a new processing route aiming at growing thick, stable, and biocompatible poly(methyl methacrylate) (PMMA) layers covalently grafted on Ti substrates. It consists of a three-step methodology (Scheme 1).

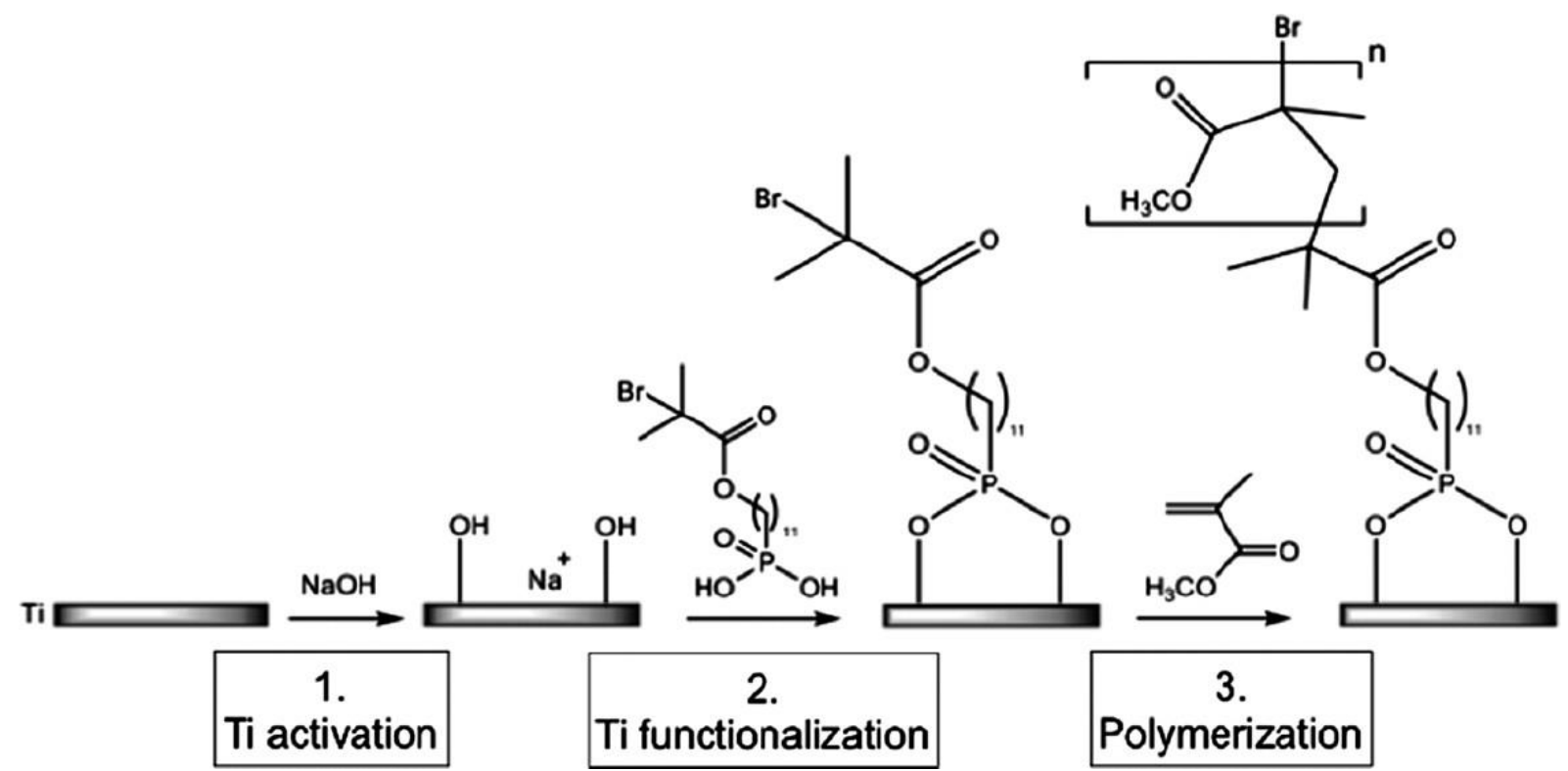

Scheme 1. Methodology used to design the PMMA-coated Ti surfaces: (1) Activation of Ti with NaOH; (2) Grafting of the bromoisobutyrate-undecyl-1-phosphonic acid Initiator; and (3) Growth of the PMMA by Surface-Initiated Atom Transfer Radical Polymerization (SI-ATRP)

First, titanium substrates are activated; second, a phosphonic acid derivative used both as a coupling agent and as a polymerization initiator is anchored on the activated substrates through a "grafting-from" method. Third, surfaceinitiated atom transfer radical polymerization (SI-ATRP) is employed to grow tethered PMMA chains. The sample surface after each step is carefully characterized; moreover, the Ti/PMMA adhesion, the durability, and the biocompatibility of the coating are evaluated. 


\section{EXPERIMENTAL SECTION}

2.1. Sample Preparation. Titanium disks ( $20 \mathrm{~mm}$ in diameter and $3 \mathrm{~mm}$ thick), cut from commercially pure grade 4 Ti bars and grade 2 Ti sheets $(20 \times 20 \times 0.5 \mathrm{~mm} 3$ and $20 \times 60 \times 0.5 \mathrm{~mm} 3)$, were used. After mechanical polishing with $600,1000,2400$, and 4000 grit silicon carbide papers, the samples were cleaned by ultrasonication in acetone, ethanol, and deionized water, $10 \mathrm{~min}$ each, to remove the residues of the mechanical treatment. The solvents were purchased from Carlo Erba.

2.2. Alkali-Activated Ti Surfaces. Ti surfaces were activated by immersing the disks for $1 \mathrm{~h}$ into a $2 \mathrm{M}$ sodium hydroxide $(\mathrm{NaOH})$ solution heated at $80{ }^{\circ} \mathrm{C}$ in a Teflon beaker.35 During the activation reaction, Ti specimens were kept suspended in the reactive media with a Teflon wire, avoiding any contact between them. These samples have been labeled as alkali-activated $\mathrm{Ti}$.

2.3. Initiator-Modified Ti Surfaces. After washing with deionized water, the alkali-activated Ti samples were suspended in a flask containing a solution of $33 \mathrm{mg}$ of synthesized bromoisobutyrateundecyl-1-phosphonic acid36 dissolved in $40 \mathrm{~mL}$ of deionized water. Stirring was maintained for $10 \mathrm{~min}$ at $55^{\circ} \mathrm{C}$ to favor the dissolution of the initiator. Then, the solution was heated up to $100{ }^{\circ} \mathrm{C}$ for $24 \mathrm{~h}$ in the dark. The grafting reaction was carried out in the darkness to avoid side reactions caused by the well-known photocatalytic activity of TiO2.37,38 At the end of the reaction, the samples were cleaned by ultrasonication in dichloromethane and deionized water, $10 \mathrm{~min}$ each, to remove the ATRP initiator only physisorbed on the metallic surface. These samples have been labeled as initiator-modified Ti.

2.4. PMMA-Coated Ti Surfaces. Initiator-modified Ti surfaces were transferred in a reactor where the ATRP of the monomer took place according to the following procedure:

(a) the reactor was equipped with a magnetic stirrer, degassed (three times; alternating between vacuum and argon), and finally filled with argon;

(b) copper bromide (39 mg, $\mathrm{Cu}(\mathrm{I}) \mathrm{Br}$ ) (Sigma-Aldrich), $9 \mathrm{~mL}$ of anisole (Acros Organics), and $48 \mu \mathrm{L}$ of pentamethylene diethyl-triamine (PMDETA) (TCI) were incorporated and stirred to yield a homogeneous solution;

(c) malononitrile (18 mg, TCI) was then included in the reactive media to enhance the polymerization rate;36 and

(d) MMA (106 mmol, Acros Organics)-purified from its stabilizer by column chromatography on basic aluminum oxide (SigmaAldrich)-was finally added in the react, and the reaction was carried out under argon at $35^{\circ} \mathrm{C}$ for 24 h. The quantity of the monomer was optimized to get the highest amount of monomer in a $20 \mathrm{~mL}$ and $5.3 \mathrm{M}$ concentrated solution.

Once the selected time was reached, the specimens were washed by ultrasound in methanol to remove the residues of $\mathrm{Cu}(\mathrm{I}) \mathrm{Br}$ and PMDETA as well as the unreacted monomer. These samples have been labeled as PMMA-coated Ti.

2.5. Chemical Characterizations. The reactions were followed by attenuated total reflection Fourier-transform infrared spectroscopy (ATR-FTIR) and X-ray photoelectron spectroscopy (XPS). The former analysis was performed using a PerkinElmer spectrometer, whereas the XPS measurements were carried out in an ultrahigh vacuum spectrometer described elsewhere.39 Spectra were recorded with a VSW class WA hemispherical electron analyzer using a nonmonochromated Al Ka X-ray source (1486.6 eV). Survey and high-resolution XP spectra were recorded in the constant pass energy mode (90 and $22 \mathrm{eV}$, respectively). The $\mathrm{C} 1 \mathrm{~s}$ peak at $284.6 \mathrm{eV}$ was used as an internal reference of the binding energy scale. Moreover, energy-dispersive X-ray (EDX) measurements were performed to investigate the sample elemental distribution using a QUANTAX EDX system embedded in a scanning electron microscope. Quantitative values of the elemental atomic percentage were evaluated by performing standardless analysis using Bruker ESPRIT software.

2.6. Morphological Characterizations. The samples' surface morphology, after each step of the procedure, was investigated by scanning electron microscopy (SEM) analysis through a CrossBeam workstation (focused ion beam (FIB)-SEM) AURIGA-Zeiss 405 microscope equipped with a QUANTAX EDX system. An atomic force microscope (Multimode, Bruker) equipped with Si cantilevers (OTESPA, Bruker) was used to reconstruct the 3D sample topographies. 
2.7. Cross-Sectional Analysis. The FIB facility (Cobra, Physics d'Orsay), incorporated in the CrossBeam workstation AURIGA-Zeiss 405 microscope, was used to obtain sample cross sections. The sample was tilted by an angle equal to $54^{\circ}$, with respect to the horizontal line, and a focused beam of gallium ions $\left(\mathrm{Ga}^{+}\right)$hits the sample surface (with an ion current of $120 \mathrm{pA}$ for $30 \mathrm{~s}$ ) which sputtered a small amount of material, producing a cut on the sample. Simultaneously, secondary electrons were also collected to form an image so that the cut of the sample was followed in real time, allowing the optimization of the beam currents to prevent the damage of the interface and to minimize the redeposition of Ga. EDX measurements were used to evaluate the cross-sectional elemental distribution. Spectra were recorded at $4 \mathrm{keV}$ both on the top view of the surface and across the interface. In particular, the variation of the relative element concentration from the bulk Ti substrate toward the polymer surface was determined by collecting 29 number of EDX spectra, each for $30 \mathrm{~s}$, along a line of $880 \mathrm{~nm}$.

2.8. Thermogravimetric Analysis. The average thickness of the PMMA layers was estimated by thermogravimetric analysis (TGA) performed with a Q50 apparatus (TA Instruments) under air at $5^{\circ} \mathrm{C} / \mathrm{min}$. The polymer was grown on a $5 \times 5 \mathrm{~mm}^{2}$ Ti sheet, with a thickness of $0.15 \mathrm{~mm}$, to be able to measure the weight loss after the polymer decomposition process. From the measured weight loss $(6.5 \mu \mathrm{g})$, the polymer thickness was calculated taking into account the titanium and the PMMA densities and the Ti foil thickness.

2.9. Adhesion Strength Evaluation. The adhesion strength between the grown PMMA layer and the underlying Ti substrate was evaluated by scratch tests. For this purpose, a sapphire spherical indenter with a radius of $1000 \mu \mathrm{m}$ was employed, and 10 scratches, under constant normal force $\left(F_{n}\right)$ using a constant speed of $30 \mu \mathrm{m} / \mathrm{s}$, were made. $\mathrm{F}_{\mathrm{n}}$ was increased at each scratch from 0.6 to $9.2 \mathrm{~N}$. After that, the SEM images of the performed scratches were used to (i) observe the contact damage, (ii) measure the residual groove widths (2r), and (iii) estimate the contact area $\left(\mathrm{A}_{\mathrm{c}}=\pi \mathrm{r}^{2}\right)$. With the values of the contact area and the applied normal force, the critical mean contact pressure $\left(\mathrm{F}_{\mathrm{n}} / \mathrm{A}_{\mathrm{c}}\right)$-namely, the load at which delamination of the polymer layer occurs-was estimated.40

2.10. Stability of PMMA-Coated Ti Soaked in Saturated Simulated Body Fluid. To evaluate the sample stability, PMMAcoated Ti samples were soaked in a 1.5x simulated body fluid (SBF) solution (e.g., a solution with ion concentrations 1.5 times higher than that of a standard c-type SBF). 41,42 The temperature was maintained at $37^{\circ} \mathrm{C}$, and the $\mathrm{pH}$ was set to $7.3 \pm 0.1$ using a titrate solution consisting of tris(hydroxymethyl)aminomethane $\left(\left(\mathrm{CH}_{2} \mathrm{OH}\right)_{3} \mathrm{CNH}_{2}\right)$ and hydrochloric acid. Three different immersion periods $(2,14$, and 21 days) were chosen. The samples' surface morphology and composition, after each soaking period, were investigated by performing SEM and FTIR analyses. The $1.5 \times$ SBF solution was refreshed once in every 7 days.

2.11. Cytotoxicity Tests. BJ2 human fibroblasts, obtained from ATCC, were used for experiments. Cells were cultured in the Eagle's minimum essential medium (ATCC) supplemented with $10 \%$ fetal bovine serum, $1 \%$ antibiotics (penicillin-streptomycin), and $0.2 \%$ fungizone at $37^{\circ} \mathrm{C}$ in a $5 \% \mathrm{CO}_{2}$ atmosphere. Human umbilical cord vascular endothelial cells were purchased from PromoCell and were cultured in an endothelial cell growth medium with endothelial cell growth mix supplementation under standard culture conditions. Prior to seeding, cells were harvested using $0.05 \%$ trypsin/ $/ 0.02 \%$ EDTA, centrifuged, suspended in the media, and used for essays. After $24 \mathrm{~h}$ and 3 and 6 days, cell viability was evaluated by performing colorimetric metabolic alamarBlue assay. Briefly, samples were incubated with $10 \%$ resazurin (fluorimetric cell viability kit I, PromoKine) in the cell culture medium for $2 \mathrm{~h}$. The reduction of resazurin, as an indirect measure of cellular metabolic activity, was assessed using manufacturer's instructions. The amount of fluorescence was monitored with a SAFAS Xenius XML fluorescence reader (SAFAS, Monaco) at an excitation wavelength of $560 \mathrm{~nm}$ and an emission wavelength of 590 $\mathrm{nm}$. The morphology of the cells cultivated directly onto the samples surface was evaluated by scanning electron microscopy (SEM) analysis. Cells were fixed with $4 \%$ glutaraldehyde, washed with phosphate-buffered saline (PBS) solution, and dehydrated using an alcohol series of increasing concentrations (50, 70, 95, and 100\%, 5 min each). Subsequently, samples were incubated in 100\% ethanol/hexamethyl disilazane (HMDS) (1:1 v/v) for $5 \mathrm{~min}$, then only in HMDS for $10 \mathrm{~min}$, and dried overnight. Samples were then mounted onto SEM stubs using a conductive carbon tape and coated with gold/palladium in a sputter coater under argon atmosphere (using a current of $7.5 \mathrm{~mA}$ for $3 \mathrm{~min}$ ). Images at various magnifications were acquired using a Hitachi TM-1000 scanning electron microscope.

2.12. Immunofluorescence. The possibility to further functionalize the grown PMMA layer with biopolymers was investigated.43 As a case of study, a dilute solution of gelatin ( $0.1 \mathrm{w} / \mathrm{v} \%$ in PBS) was deposited by adsorption on the PMMA for $1 \mathrm{~h}$ at room temperature (RT) followed by copious washing with PBS, and its effect on the cell behavior was assessed by immunofluorescence staining. Visualization of F-actin filaments was done using fluorophore-conjugated phalloidin (Alexa-Fluor 568, Invitrogen). In particular, cells were fixed with $4 \%$ 
paraformaldehyde in PBS for 10 min at RT. Then, they were washed twice with PBS and permeabilized with $0.2 \%$ Triton X-100 for $5 \mathrm{~min}$ at RT and again washed with PBS twice. Successively, cells were incubated with $1 \%$ bovine serum albumin in PBS for 20 min prior to the addition of phalloidin. Phalloidin was added, and the cells were incubated for $30 \mathrm{~min}$ at RT in the dark. Subsequently, DAPI (PromoCell) was added to label the nuclei for 5 min in the dark at RT. Then, the cells were washed in PBS to remove the unbound phalloidin and DAPI, and the samples were kept in PBS for imaging. Fluorescence images were captured using Nikon Eclipse Ti-S with 10x and 40x objectives equipped with a Nikon digital camera (equipped with NIS-Elements software).

\section{RESULTS AND DISCUSSION}
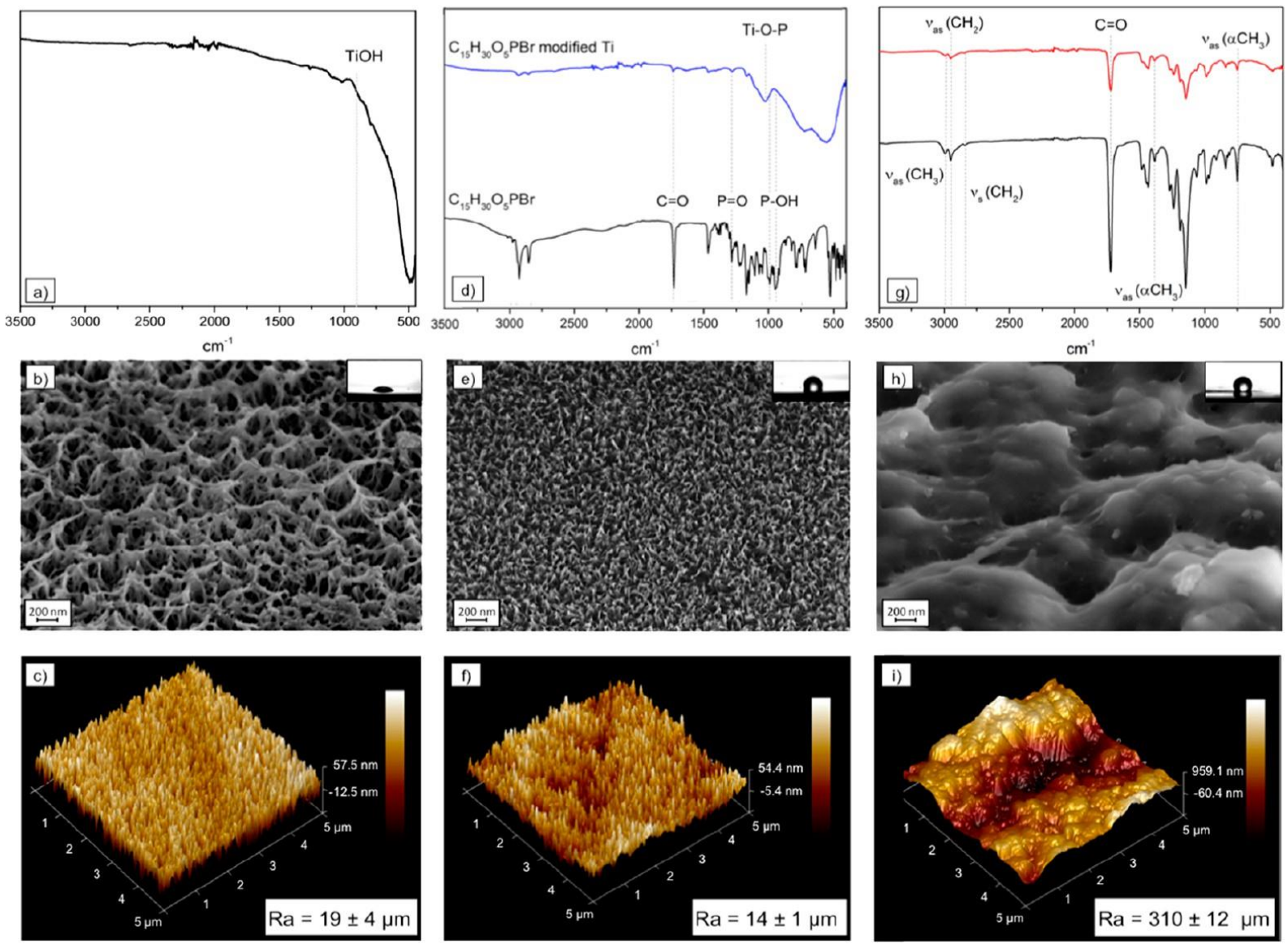

Figure 1. (a) ATR-FTIR spectra of the alkali-activated Ti surface; (b) SEM image of the alkali-activated Ti surface (inset: contact angle measurement); (c) atomic force microscopy (AFM) 3D reconstruction of the alkali-activated Ti surface topography (scan size $\left.5 \times 5 \mu \mathrm{m}^{2}\right)$; (d) ATR-FTIR spectra of the bromoisobutyrate-undecyl-1-phosphonic acid polymerization initiator $(\mathrm{C} 15 \mathrm{H} 30 \mathrm{O} 5 \mathrm{PBr}$, black curve) and of the initiatormodified Ti surface initiator (blue); (e) SEM micrograph of the initiator-modified surface (inset: contact angle measurement); (f) AFM 3D reconstruction of the initiator-modified Ti surface (scan size $5 \times 5 \mu \mathrm{m}^{2}$ ); (g) comparison between the ATR-FTIR spectra of a PMMA powder (black) and that of the grown PMMA layer (red); (h) SEM micrograph of the PMMA-coated Ti surface (inset: contact angle measurement); and (i) AFM 3D reconstruction of the PMMA-coated Ti surface (scan size $5 \times 5 \mu \mathrm{m}^{2}$ ).

This work reports an original methodology based on the preactivation of the Ti substrates in a basic media to create surface hydroxyl sites.44 This alkali activation leads to a hierarchical microporous interlayer, which acts as a scaffold for the following polymerization reaction. It has a double functionality: (i) promote the grafting reaction because of high hydroxyl groups' content and (ii) drive the growth of the polymer chains along the pillared structure avoiding their collapse. This methodology allows the growth of thick PMMA layers covalently bound on Ti flat substrates via SI-ATRP using phosphonic acids as coupling agents (see Scheme 1). In particular, bromoisobutyrate-undecyl-1-phosphonic acid, already employed to grow polystyrene chains on $\mathrm{TiO}_{2}$ nanoparticles45 was used as a polymerization initiator. It contains at the one end a phosphonic acid group that links covalently onto the oxide surface and at the other end an $\alpha$-bromo ester group as an ATRP initiator. The anchorage involves the interaction between the $\mathrm{P}-\mathrm{OH}$ of the phosphonic acid and the surface hydroxyl groups $\mathrm{Ti}-\mathrm{OH}$ to form $\mathrm{Ti}-\mathrm{O}-\mathrm{P}$ bonds.33 The 11-hydrocarbon spacer used to separate the phosphonate group from the reactive one ensures the stability of the molecule. 34 To the best of our knowledge, the combination between the 
alkali activation of Ti substrates and the SI-ATRP, which allows a better control of the chain length and composition,22,46,47 has never been used to functionalize metal substrates and design a hybrid organic-inorganic nanostructure that possesses both a covalent and an interlocking nature. In particular, the following three-step methodology was performed (Scheme 1)
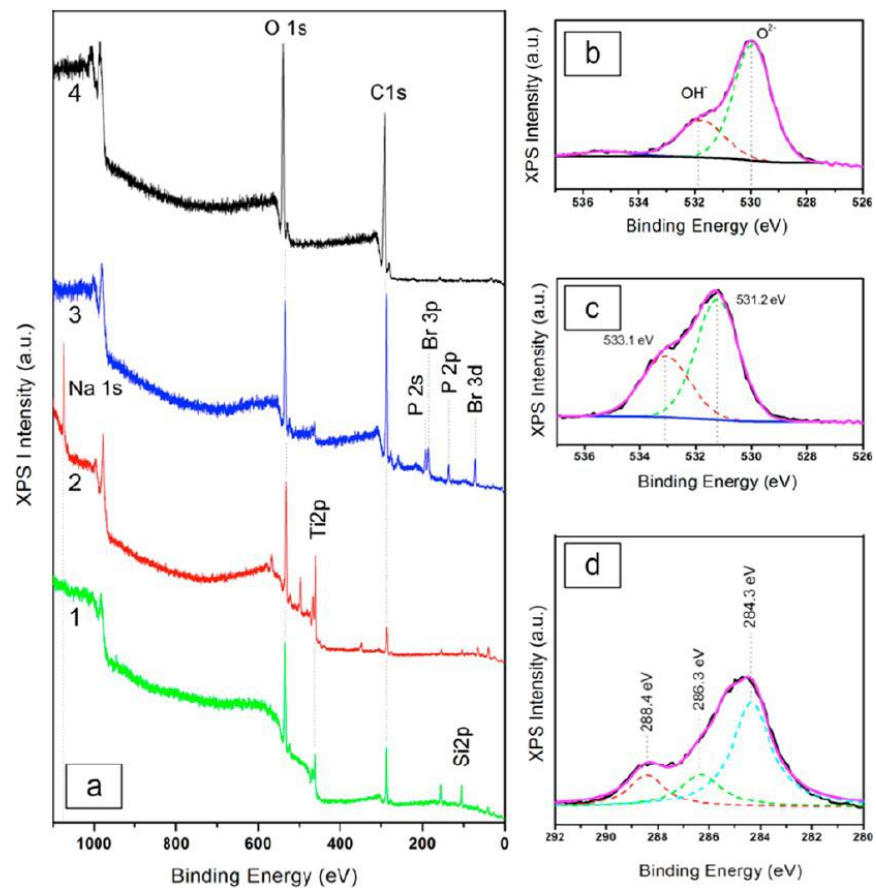

Figure 2. (a) XPS survey spectra of a polished Ti substrate (green, 1), activated Ti sample (red, 2), initiator-modified Ti (blue, 3), and PMMA-coated Ti (black, 4); (b) XPS narrow-scan spectrum of the O 1s peak of the alkali-activated Ti surfaces; (c) XPS spectrum of the $\mathrm{O}$ 1s peak of the initiator-modified Ti surfaces; and (d) $\mathrm{C}$ 1s peak of the PMMA-coated Ti samples.

3.1. Alkali-Activated Ti Surfaces. The aim of the activation treatment was to create active sites on the surface where the phosphonic acid could anchor.33 The reaction was followed by ATR-FTIR, the spectrum of which is reported in Figure 1a. The absorption band in the range 800 and $500 \mathrm{~cm}^{-1}$ is attributable to the Ti-O bonds generated by the native oxide, sodium oxide, and the surface hydroxide, whereas the shoulder around $950 \mathrm{~cm}^{-1}$ can be attributed to $\mathrm{Ti}-\mathrm{OH}$ bonds. 48 Previous works have shown that an alkali treatment leads to the formation of a microporous layer of $\mathrm{Na}_{2} \mathrm{Ti}_{5} \mathrm{O}_{11} .49$ Indeed, during the alkali treatment, the $\mathrm{TiO}_{2}$ native oxide layer, which covers and passivates the Ti surface, partially dissolves in the alkali solution because of the attack by hydroxyl groups.50 This mechanism involves local cathodic and anodic reactions. The former leads to hydration of Ti with the generation of $\mathrm{H}_{2}$, partially adsorbed in the near-surface region of the Ti substrates. The latter produces negatively charged and hydrate substrates which are able to combine with the sodium ions and form a porous sodium titanate layer.51 To establish an interconnected microporous layer, the $\mathrm{NaOH}$ concentration and the time of reaction were dramatically reduced with respect to the previous works.52,53 Its pore size ranges between 50 and $200 \mathrm{~nm}$, and it is uniformly spread on the entire surface, as shown in Figure 1b. The layer consists of pillared and coneshaped structures, making the surface hydrophilic with a contact angle of $25 \pm 9^{\circ}$ (see the inset of Figure 1b). Moreover, according to the AFM images (Figure 1c), an average roughness of $19 \pm 4 \mathrm{~nm}$ was determined. Furthermore, no diffraction lines, except those of the Ti substrates, were detected in the X-ray diffraction pattern, indicating that the microporous layer is amorphous.

3.2. Initiator-Modified Ti Surfaces. The anchorage of the initiator on the alkali-activated Ti surfaces was followed by ATR-FTIR whose spectrum is reported in Figure 1d. In particular, comparison between the ATR-FTIR spectrum of the employed initiator (black) and that of the initiator-modified Ti surfaces (blue line) confirms that the initiator was successfully grafted on the activated Ti substrate. Indeed, in accordance with the previous studies, 16,45 the attenuation of the $\mathrm{P}-\mathrm{O}-\mathrm{H}$ stretching vibration peaks (in the range of $(940-100) \mathrm{cm}^{-1}$ ) and the $\mathrm{P}=\mathrm{O}$ peaks $(1270 \mathrm{~cm}-1)$ together with the simultaneous presence of the band at $1028 \mathrm{~cm}^{-1}$, assigned to the formation of $\mathrm{Ti}-\mathrm{O}-\mathrm{P}$ bonds, indicates that the organic initiator was covalently grafted preferentially via the formation of tridentate bonding modes. The successful grafting was also confirmed by the XPS analysis (Figure 2a), whose spectrum (blue line (3)) displays the elemental peak characteristics of the initiator molecule: $\mathrm{O} 1 \mathrm{~s}$ 
(531.8 eV), C 1s (284.5 eV), P 2s (192 eV), Br 3p (185 eV), P 2p (134 eV), and Br 3d (72 eV). As expected, P and $\mathrm{Br}$ signals were not detected in case of in the bare titanium (green line (1)) and in the alkali-activated Ti surfaces (red line (2)). Whereas the Si 2p peak, observed on the bare titanium (green line (1), Figure 2a), is due to slight contamination with silicon generated during the Ti mechanical polishing. Moreover, the Na 1s peak (at $1071 \mathrm{eV}$ ) visible after the activation step disappeared after the grafting reaction, suggesting that the Na atoms initially incorporated into the microporous layer were released in the solution during the grafting reaction. This latter assumption is in agreement with the inductively coupled plasma atomic emission spectroscopy (ICP-AES) analysis, which detects $\mathrm{Na}$ atoms in the grafting residual solution. Moreover, the investigation of the asymmetrical $\mathrm{O}$ 1s peak (Figure 2c) confirms the covalent adsorption of the initiator molecules at the Ti surfaces. Indeed, the $\mathrm{O}$ 1s peak can be deconvoluted into two symmetric components centered at 531.2 and $533.1 \mathrm{eV}$. The former is assigned to $\mathrm{Ti}-\mathrm{O}-\mathrm{P}$ bonds established between the $\mathrm{P}-\mathrm{OH}$ and the surface hydroxyl groups (in Figure $2 \mathrm{~b}$ ), and the latter is assigned to the $\mathrm{P}-\mathrm{O}-\mathrm{H}$ bonds.54,55 Thus, it can be pointed out that the grafting reaction leads to (i) a change of the surface composition and (ii) a reorganization of the surface morphology. Indeed, as depicted in Figure 1e, this latter consists of a more compact and pointed organic pillared structure with a decreased surface wettability (contact angle equal to $97 \pm 3^{\circ}$ ). Furthermore, the slight decrease of the surface roughness $(\mathrm{Ra}=14 \pm$ $1 \mathrm{~nm}$, Figure 1f) suggests that the organic initiator is attached inside the pores left by the activation step. To evaluate the spatial distribution of the initiator molecules on the surface, high-resolution maps of the bromine concentration were reconstructed (Figure 3). It has been pointed out that bromine was almost uniformly spread all over the surface with an atomic percentage between 0.4 and $1.2 \%$.

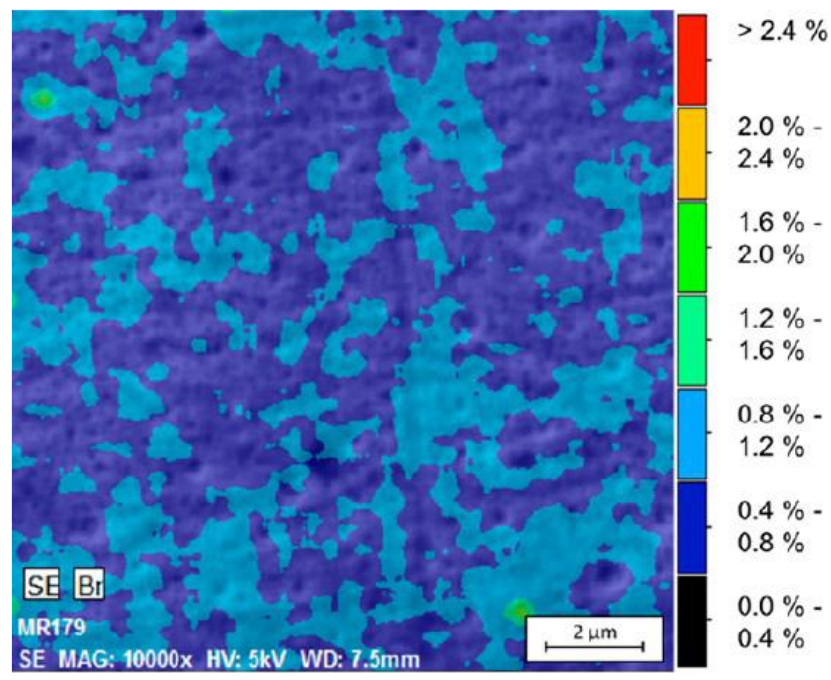

Figure 3. EDX high-resolution map of the bromine atomic percentage surface distribution

3.3. PMMA-Coated Ti Surfaces. During the ATRP reaction, the active species (or radicals) are generated by a reversible oxidation-reduction reaction catalyzed by a transition-metal complex (i.e., the ATRP catalyst). Its function is to produce active species on which the monomer can be added. The transition metal complex - selected in this work - results from the in situ addition of $\mathrm{Cu}(\mathrm{I}) \mathrm{Br}$ and PMDETA at a $[\mathrm{Cu}(\mathrm{I}) \mathrm{Br} / \mathrm{PMDETA}]$ molar ratio equal to 1 . Because the accessibility of the initiator immobilized on rough surfaces decreases with respect to flat and smooth substrates, a large excess of catalysts, in regard to the grafted initiator, was used. In addition, to increase the thickness of the polymer layer, malononitrile, known to enhance the polymerization rate,53 was added in the same concentration as copper bromide. Such a property of malononitrile was previously used for the ATRP of polystyrene on titanium oxide nanoparticles.45 Finally, the concentration of the monomer was also optimized and equal to $5.3 \mathrm{~mol} / \mathrm{L}$. FTIR spectra of the obtained surface-confined PMMA chains (red curve) and synthesized PMMA powders (black curve) are shown in Figure 1g. They both contain the carbonyl group peak $(\mathrm{C}=\mathrm{O})$ and the $\alpha$-methyl group vibration $\left(\alpha-\mathrm{CH}_{3}\right)$ signal, confirming that the PMMA layer was successfully grown. In more detail, the spectrum of the PMMA chains reveals the presence of three peaks in the $2800-3000 \mathrm{~cm}^{-1}$ region: the characteristic peaks at 2924 and $2852 \mathrm{~cm}^{-1}$ corresponding to asymmetric and symmetric stretching vibrations of the methylene groups $\left(\mathrm{CH}_{2}\right)$ and the broad band corresponding to the methyl asymmetric stretch $v$ as $\left(\mathrm{CH}_{3}\right)$ at 2960 $\mathrm{cm}^{-1}$. The sharp peak at $1730 \mathrm{~cm}^{-1}$ is assigned to the carbonyl group stretching.56 Whereas the two bands at 1382 and $747 \mathrm{~cm}^{-1}$ are attributed to the $\alpha$-methyl group vibrations.56 Moreover, in the survey XPS spectra of the polymer film (black line (4), Figure 2a), only the intense peaks of $\mathrm{C} 1$ s at $284.6 \mathrm{eV}$ and $\mathrm{O} 1 \mathrm{~s}$ at $531.8 \mathrm{eV}$ are 
visible. This indicates that the grown rounded polymer layer is dense without cracks and covers the entire surface, as shown in the SEM images (Figure 1h). The analysis of the C 1s peak, in Figure 2d, highlights the presence of three peaks, which correspond to the different bonds involving the carbon atoms of the PMMA chain. In particular, the peak centered at $284.6 \mathrm{eV}$ is attributed to both the $\mathrm{C}-\mathrm{C}$ and $\mathrm{C}-\mathrm{H}$ bonds, whereas the last two are assigned to the $\mathrm{C}-\mathrm{O}$ bonds (at $286.7 \mathrm{eV}$ ) and to the presence of the carbonyl groups $\mathrm{C}=\mathrm{O}$ (at 288.4 eV).57 The absence of peaks related to the Ti substrate is due to the thickness of the organic layer, which is higher than the photoelectron escape depth ( $\sim 5 \mathrm{~nm}$ in the experimental condition used). Furthermore, an increase of the average roughness Ra $=310 \pm 12 \mathrm{~nm}$ (AFM topography, Figure 1i) and a slight reduction of the surface wettability (contact angle $104 \pm$ $8^{\circ}$, inset Figure 1h) were observed. It can be stated that our strategy allows the fabrication of PMMA-coated Ti systems with size ranging from 400 to $1200 \mathrm{~mm}^{2}$ and an average PMMA layer thickness of $1.9 \pm 0.3 \mu \mathrm{m}$, estimated by TGA (Figure $\mathrm{S} 1$ in the Supporting Information).
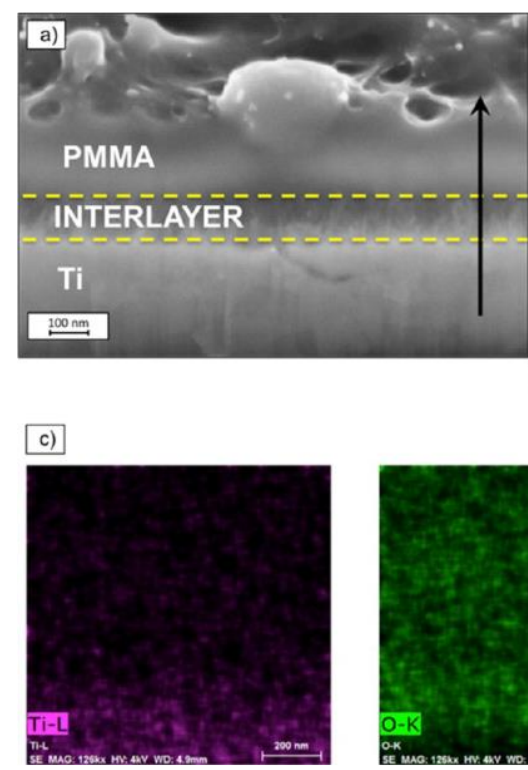

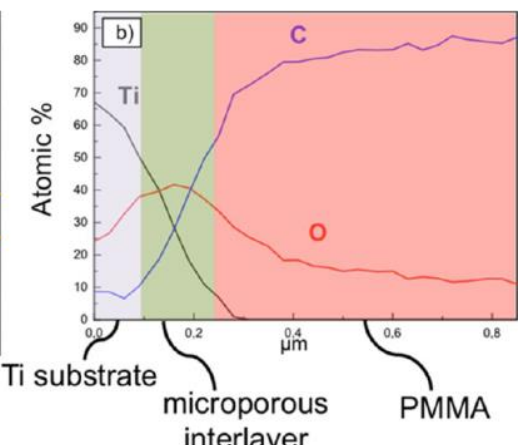
interlayer

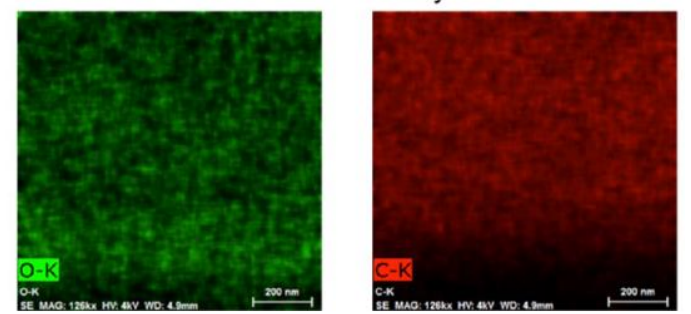

Figure 4. (a) SEM image of the PMMA-coated Ti cross section obtained by the FIB technique; (b) element concentration along the cross line (black arrow) obtained by EDX analysis; and (c) EDX maps showing, from left to right, Ti (violet), oxygen (green), and carbon (red).

3.4. Cross-Sectional Analysis. Cross sections of the PMMA-coated Ti were obtained using FIB-SEM techniques (Figure 4a). Three areas are visible: at the bottom Ti and its columnar structure, in the middle the microporous interlayer above $\mathrm{Ti}$, and at the top the polymer. Local and global EDX analyses were also performed to highlight the variation and spatial distribution of the cross-sectional elemental composition. In particular, the variation of the relative element concentration from the Ti substrate toward the polymer surface, according to the black arrow depicted in Figure 4a, is shown in Figure 4b. The $\mathrm{C}$ content increases and simultaneously the Ti content decreases in the interlayer, and the $\mathrm{O}$ signal reaches a maximum in the middle of the interlayer and then decreases to a constant value in the polymer layer. EDX maps (Figure 4c) focus on the spatial distribution of Ti (violet), O (green), and $\mathrm{C}$ (red) elements. Ti and polymer zones are easily distinguishable in $\mathrm{Ti}$ and $\mathrm{C}$ mapping, whereas the $\mathrm{O}$ signal is detected in the interlayer and in the polymer, as already highlighted by the cross line (Figure 4b). These variations are in agreement with the strong interpenetration of the polymer inside the microporous layer created by the alkali treatment of the Ti substrate. The covalent link between the organic part and the metallic one is proven by the Ti-O-P band visible in the ATR-FTIR spectra of the initiator-modified Ti and PMMA-coated Ti. Moreover, as better highlighted in our previous work,58 the Ti/PMMA interface is characterized by an underneath porosity which soften the PMMA layer, reducing its Young's modulus with respect to the commercial bulk PMMA. In addition, to study the effect of adding the malononitrile in the ATRP reactive media, the cross section of a PMMA-coated Ti interface grown without malononitrile was observed. As evidenced by the micrograph reported in Figure 5, the polymer layer is less compact and thinner than the one grown in the presence of malononitrile, confirming that it enhances the reaction rate leading to a higher polymer thickness (see Figure 4a).

3.5. Adhesion Strength Evaluation. The adhesion strength between the grown PMMA layer and the underlying Ti substrate was evaluated by performing scratch tests. Low force ( $\mathrm{Fn}=0.6 \mathrm{~N}$, Figure 6a) results in an indenter-sample discontinuous contact. Indeed, the tip is in contact with only the higher surface asperities; by 
increasing the force, the contact becomes continuous ( $\mathrm{Fn}=1.6 \mathrm{~N}$, Figure $6 \mathrm{~b}$ ). At this force level, it is possible to observe that the coalescence of those surface topography irregularities generates a cracklike pattern in the region where two consecutive asperities collapse together. This cracklike pattern seems to be oriented perpendicularly to the indenter displacement (Figure 6b). For Fn $=3.5 \mathrm{~N}$, tensile stresses, 59 acting at the end of the tip-sample contact, lead to the delamination of the coating from the substrate (Figure 6c). This load is identified as the critical load of the grown PMMA layers; taking into account the tip-sample contact area in this region $(2 \mathrm{r}=130 \mu \mathrm{m})$, a critical stress of $260 \mathrm{MPa}$ can be estimated (fully circular contact was chosen because of the thin thickness of the PMMA layer and the elastic behavior of the Ti substrate). By increasing the load, the delaminated area also augments, overreaching the edge of the groove (Figure 6d,e). Nevertheless, in the range of 4.5-6.4 N, delamination is not totally achieved; many regions of coating can be still observed in the scratch groove. It is necessary to reach more than $9 \mathrm{~N}$ to get the gross spallation of the coating59 (Figure 6f). This good Ti/PMMA adhesion arises from the synergic growth of polymer within the porosity of the microporous layer, and the covalent link is established through the grafting of the initiator.

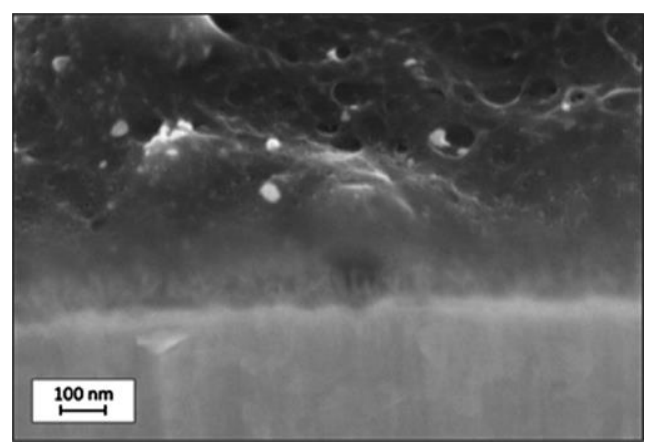

Figure 5. SEM image of the polymer layer grown without adding malononitrile in the ATRP reactive media.

3.6. Stability of PMMA-Coated Ti Soaked SBF. Because human body fluids may represent an aggressive environment for implantable materials,60 an investigation of its influence on the PMMA-coated Ti surface properties was performed. In the presence of the physical and chemical conditions of the human body, the coating could lose its stability and may corrode or degrade.55 Morphological and compositional surface properties of the PMMA-coated Ti were assessed after soaking the specimen in a SBF solution.40 The evolution of the morphology and composition of the sample surface after each soaking period was checked by performing SEM and FTIR analyses, respectively. FTIR spectra and SEM micrographies are shown in Figure 7.

No significant variation was revealed, and the typical features of the polymer morphology are still visible. Moreover, as highlighted in the FTIR spectra, both peaks related to the carbonyl group and the bands ascribed to the $\alpha$-methyl group vibrations are well-distinguishable even after a soaking period of 21 days (blue curve), proving the good stability of the PMMA layers.
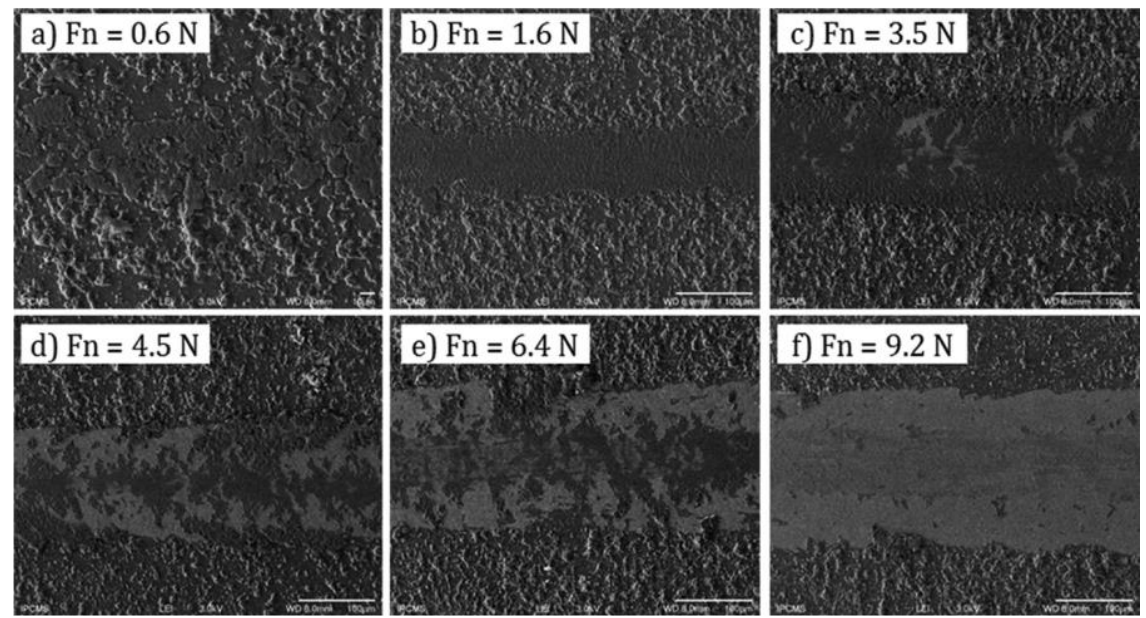

Figure 6. SEM micrographs of scratch tests on PMMA-coated Ti samples performed with an indenter of $1000 \mu \mathrm{m}$ of radius with an applied force of $0.6(\mathrm{a}), 1.6(\mathrm{~b}), 3.5(\mathrm{c}), 4.5$ (d), $6.4(\mathrm{e})$, and $9.2 \mathrm{~N}(\mathrm{f})$. Scale bar: $100 \mu \mathrm{m}$. 


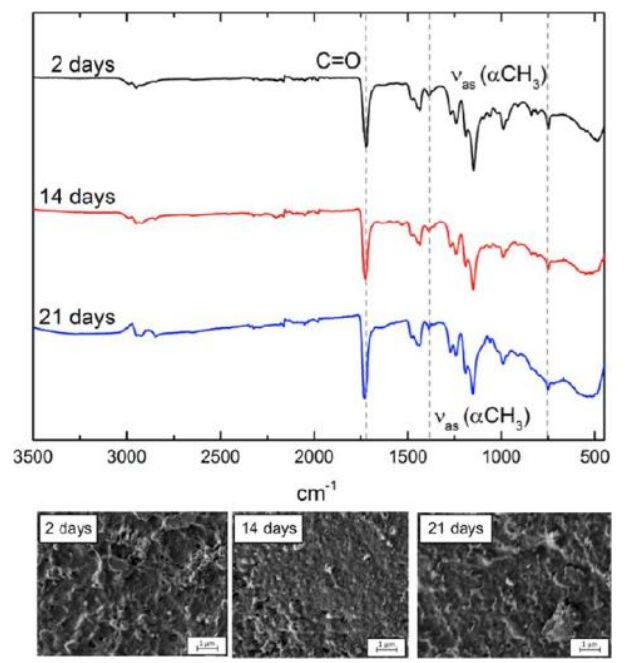

Figure 7. Top: FTIR spectra of the PMMA-coated Ti samples soaked in a 1.5× SBF solution for 2 days (black curve), 14 days (red), and 21 days (blue). Bottom: SEM images of the sample surface morphology after each soaking period.

3.7. Cytotoxicity Evaluation. Finally, to demonstrate the biocompatibility of the PMMA layer, human fibroblasts and endothelial cells were seeded and let proliferate on grown PMMA-modified Ti and on untreated Ti substrates (used as control samples). The cells morphology and growth on PMMA-coated Ti interface were compared with the one grown on pure Ti. It has been shown by alamarBlue assay that the fibroblasts grew on the modified surface over a course of 6 days (see the increase of the cell fluorescence intensity after 1 day and 6 days, red histogram in Figure 8). Although the presence of PMMA resulted in less cell numbers, compared to that obtained on the untreated $\mathrm{Ti}$ (blue histogram in Figure 8) for fibroblasts, the overall cell behavior suggests that PMMA does not detrimentally affect their growth. Indeed, it was observed that the fibroblasts were able to adhere and spread on both the untreated (Figure 9a,c) and the PMMA-coated Ti substrates (Figure 9b,d). Furthermore, the areas richer in PMMA promoted the formation of cell clusters and cells with multiple filopodia (Figure 9d). However, the initial attachment of human umbilical cord vascular endothelial cells (HUVEC) on modified Ti surfaces was higher than that on the untreated Ti surfaces, as observed microscopically and from the metabolic activity (Figure 10). Moreover, the morphology and behavior of the cells ensure that the PMMA modification will have no detrimental effect on the implant surrounding the tissue interface. For direct tissue-contact applications, the PMMA-coated Ti could be further functionalized with natural biopolymers (e.g., gelatin). As a proof of concept, a dilute solution of gelatin $(0.1 \mathrm{w} / \mathrm{v} \%)$ was deposited onto the untreated Ti and PMMAcoated Ti and its effect was investigated by immunofluorescence staining. Figure 11 illustrates the fluorescence images of the cells grown on the unmodified $\mathrm{Ti}$ (Figure 11a) and on the gelatin-modified Ti (Figure 11c) and those of the PMMAcoated Ti samples (not modified in Figure 11b and functionalized with gelatin in Figure 11d). It was observed that the PMMA-coated Ti surfaces modified with gelatin result in clustering of cells (Figure 11d). This proves that the grown PMMA chains have no cytotoxic effect and that the PMMA can also be further functionalized by adsorption of additional natural polymeric components (such as gelatin). Instead of gelatin, utilization of human serum can provide a rich source of adhesive proteins such as fibronectin or vitronectin and also growth factors. By preactivation of PMMA surfaces with plasma treatment, the level of adsorption can be significantly increased. For more long-term and robust functionalization, covalent linking of proteins or short peptide sequences (such as RGD) onto the grown PMMA surface can be envisioned.

\section{Metabolic activity of BJ2s}

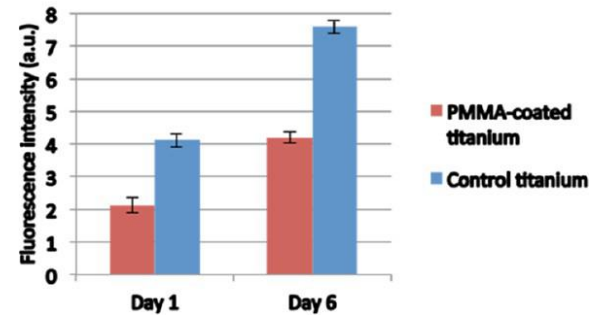

Figure 8. Metabolic activity of the BJ2 human fibroblast let proliferate on PMMA-coated Ti surfaces (red) and on uncoated Ti substrates (blue) over a period of 6 days (alamarBlue assay). 

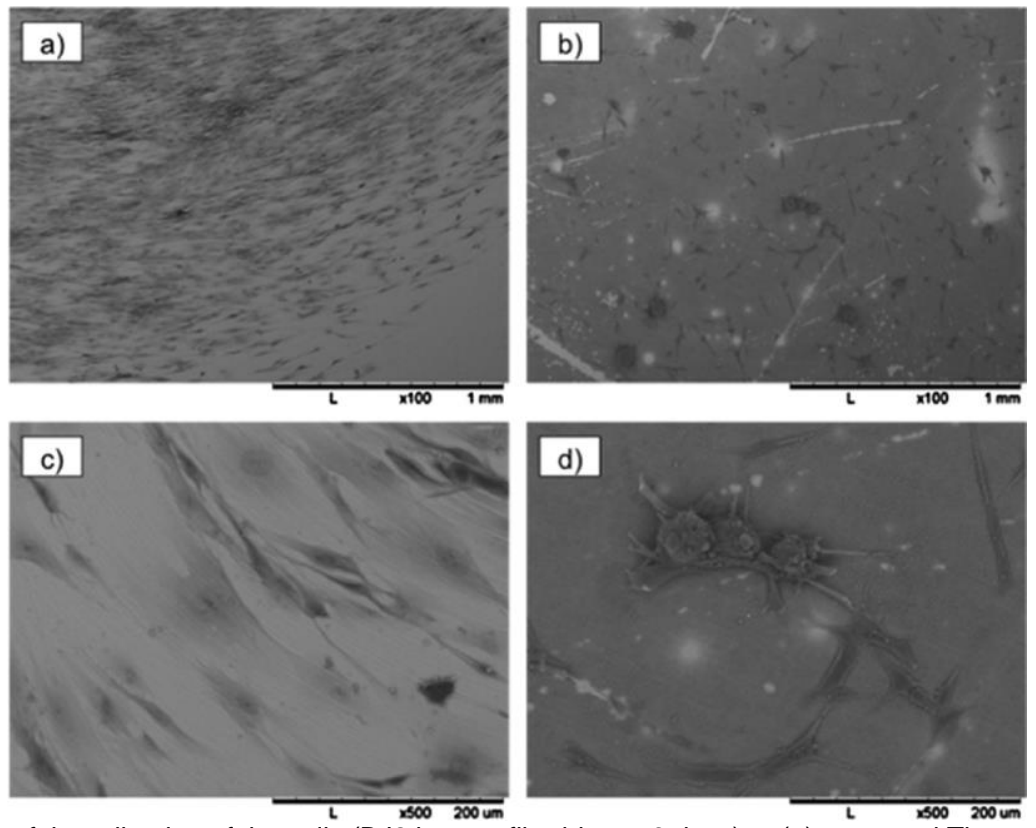

Figure 9. SEM images of the adhesion of the cells (BJ2 human fibroblasts, 6 days) to (a) untreated Ti sample (control); (b) PMMAcoated Ti substrates; and (c,d) higher magnification of $(a, b)$, respectively.

\section{CONCLUSIONS}

Overall, a strategy to grow up to 1-2 $\mu$ m of PMMA layer on the Ti surface in which the interlayer is biocompatible and the link between the polymer and the Ti possess both a covalent and an interlocking nature has been developed. The ability to reach polymer thicknesses that are significantly higher than those of other methodologies arises from the soft alkali treatment and the use of malononitrile, which represents the key elements of our approach. Indeed, the alkali treatment leads to the formation of a hierarchical porous interlayer consisting of regular pillared and cone-shaped structures with a dual function. First, it promotes the grafting reaction because of the induced-surface high hydroxyl groups' content. Second, it drives the growth of the polymer chains along the pillared structure avoiding their collapse. Moreover, the simultaneous presence of malononitrile in the reactive media further enhances the development of the polymer thickness. The synergic growth of the polymer within the porosity of the microporous layer and the covalent link established through the grafting of the initiator allows elaborating a compact graduated structure possessing high Ti/PMMA adhesion strength ( 260 MPa). Furthermore, the PMMAcoated Ti biocompatibility was proven by the attachment and spreading of human fibroblasts and endothelial cells. Besides, the chemical composition and surface morphology of the grafted PMMA remain unaltered after an incubation of 21 days in a supersaturated SBF solution, confirming its good stability. This methodology opens new opportunities toward hybrid prosthesis with adjustable mechanical properties with respect to host bone properties for personalized medicines. Moreover, the versatility of this methodology allows easy growth of polymers of polymethacrylates or polyacrylates series bonded on titanium substrates. Further works in this direction are in progress.

\section{- ASSOCIATED CONTENT}

The Supporting Information is available free of charge on the ACS Publications website at DOI: 10.1021/acsami.7b17008. Evaluation of the PMMA average thickness by performing TGA experiments (PDF)

\section{- AUTHOR INFORMATION}

Corresponding Authors: *E-mail: daniele.passeri@uniroma1.it (D.P.).

*E-mail: genevieve.pourroy@ipcms.unistra.fr (G.P.).

ORCID: Spyridon Zafeiratos: 0000-0001-8165-2585 Daniele Passeri: 0000-0001-8189-1359 Nihal Engin Vrana: 0000-0002-5398-6710

Author Contributions: The manuscript was written through contributions of all authors.

Funding:

This work has been benefitted from the financial support of the MICA Carnot Institute and the "NANOTRANSMED" project cofunded by the European Regional Development Fund (ERDF) in the framework 
of the INTERREG V Upper Rhine program “Transcending borders with every project”. Moreover, we are grateful for the financial support given by the DFG within the project PA 837/44-1 "Titanium/polymer sheets designed for biomedical application-Feasibility study". The IdEx 2016 program for "Post-doctorants" of the University of Strasbourg is acknowledged for the support in the framework of the "Titanium/polymer sheets designed for biomedical applications" (BMM/PN/AM/ 2016-096b) project.

Notes: The authors declare no competing financial interest.

\section{- ACKNOWLEDGMENTS}

The authors gratefully thank the MEB-CRO platform and J. Faerber for SEM observations of the scratches; Dr. F. Mura for the SEM-FIB characterizations; Dr. B. Heinrich for the TGA analysis; E. Voirin for the ATRP initiator synthesis; Muller and Dr. Barthes for cell culture experiments, as well as N. Beyer and Dr. Sebastian Kriegel for the help in the sample preparation.

\section{- REFERENCES}

(1) Michiardi, A.; Helary, G.; Nguyen, P.-C. T.; Gamble, L. J.;' Anagnostou, F.; Castner, D. G.; Migonney, V. Bioactive Polymer Grafting Onto Titanium Alloy Surfaces. Acta Biomater. 2010, 6, 667-675.

(2) Raynor, J. E.; Capadona, J. R.; Collard, D. M.; Petrie, T. A.; García, A. J. Polymer Brushes and Self-assembled Monolayers: Versatile Platforms to Control Cell Adhesion to Biomaterials (Review). Biointerphases 2009, 4, FA3-FA16.

(3) Ferraris, S.; Spriano, S. Antibacterial Titanium Surfaces for Medical Implants. Mater. Sci. Eng., C 2016, 61, 965-978.

(4) Godoy-Gallardo, M.; Wang, Z.; Shen, Y.; Manero, J. M.; Gil, F. J.; Rodriguez, D.; Haapasalo, M. Antibacterial Coatings on Titanium Surfaces: A Comparison Study Between in Vitro Single-species and Multispecies Biofilm. ACS Appl. Mater. Interfaces 2015, 7, 5992-6001.

(5) Huang, J.; Murata, H.; Koepsel, R. R.; Russell, A. J.; Matyjaszewski, K. Antibacterial Polypropylene via Surface-Initiated Atom Transfer Radical Polymerization. Biomacromolecules 2007, 8, 1396-1399.

(6) Godoy-Gallardo, M.; Mas-Moruno, C.; Yu, K.; Manero, J. M.; Gil, F. J.; Kizhakkedathu, J. N.; Rodriguez, D. Antibacterial Properties of hLf1-11 Peptide onto Titanium Surfaces: A Comparison Study Between Silanization and Surface Initiated Polymerization. Biomacromolecules 2015, 16, 483-496.

(7) Ionov, L.; Synytska, A.; Kaul, E.; Diez, S. Protein-Resistant Polymer Coatings Based on Surface-Adsorbed Poly(aminoethyl methacrylate)/Poly(ethylene glycol) Copolymers. Biomacromolecules 2010, 11, 233-237.

(8) Tejero, R.; Anitua, E.; Orive, G. Toward the Biomimetic Implant Surface: Biopolymers on Titanium-based Implants for Bone Regeneration. Prog. Polym. Sci. 2014, 39, 1406-1447.

(9) Ridzwan, M. I. Z.; Shuib, S.; Hassan, A. Y.; Shokri, A. A.; Ibrahim, M. N. M. Problem of Stress Shielding and Improvement to the Hip Implant Designs: A review. J. Med. Sci. 2007, 7, 460-467.

(10) Helary, G.; Noircle' re, F.; Mayingi, J.; Migonney, V. A New Approach to Graft Bioactive Polymer on Titanium Implants: Improvement of MG 63 Cell Differentiation onto this Coating. Acta Biomater. 2009, 5, 124-133.

(11) Chouirfa, H.; Migonney, V.; Falentin-Daudre, C. Grafting' Bioactive Polymers onto Titanium Implants by UV Irradiation. RSC Adv. 2016, 6, 13766-13771.

(12) Alageel, O.; Abdallah, M.-N.; Luo, Z. Y.; Del-Rio-Highsmith, J.; Cerruti, M.; Tamimi, F. Bonding Metals to Poly(methyl methacrylate) using Aryldiazonium Salts. Dent. Mater. 2015, 31, 105-114.

(13) Rieger, E.; Dupret-Bories, A.; Salou, L.; Metz-Boutigue, M.-H.; Layrolle, P.; Debry, C.; Lavalle, P.; Vrana, N. E. Controlled Implant/ Soft Tissue Interaction by Nanoscale Surface Modifications of 3D porous titanium implants. Nanoscale 2015, 7, 9908-9918.

(14) Debry, C.; Vrana, N. E.; Dupret-Bories, A. Implantation of an Artificial Larynx after Total Laryngectomy. N. Engl. J. Med. 2017, 376, 97-98. 
(15) Shimizu, K.; Malmos, K.; Hjarbæk Holm, A.; Uttrup Pedersen, S.; Daasbjerg, K.; Hinge, M. Improved Adhesion Between PMMA and Stainless Steel Modified with PMMA Brushes. ACS Appl. Mater. Interfaces 2014, $6,21308-21315$.

(16) Minet, I.; Delhalle, J.; Hevesi, L.; Mekhalif, Z. Surface-initiated ATRP of PMMA, PS and diblock PS-bPMMA copolymers from stainless steel modified by 11-(2-bromoisobutyrate)-undecyl-1-phosphonic acid. J. Colloid Interface Sci. 2009, 332, 317-326.

(17) Parfitt, G. D.; Rochester, C. H. Adsorption from Solution at the Solid/Liquid Interface; Academic Press: London, 1983; pp 105-152.

(18) Jones, R. A. L. Polymers at Surfaces and Interfaces; Cambridge University Press, 1999.

(19) Matsuda, Y.; Yanagida, H.; Ide, T.; Matsumura, H.; Tanoue, N. Bond Strength of Poly(methyl methacrylate) Denture Base Material to Cast Titanium and Cobalt-chromium Alloy. J. Adhes. Dent. 2010, 12, 223-229.

(20) Sun, C.; Zhou, F.; Shi, L.; Yu, B.; Gao, P.; Zhang, J.; Liu, W. Tribological Properties of Chemically Bonded Polyimide Films on Silicon with Polyglycidyl Methacrylate Brush as Adhesive Layer. Appl. Surf. Sci. 2006, 253, 1729-1735.

(21) Shimizu, K.; Malmos, K.; Spiegelhauer, S.-A.; Hinke, J.; Holm, A. H.; Pedersen, S. U.; Daasbjerg, K.; Hinge, M. Durability of PEEK Adhesive to Stainless Steel Modified with Aryldiazonium Salts. Int. J. Adhes. Adhes. $2014,51,1-12$.

(22) Barbey, R.; Lavanant, L.; Paripovic, D.; Schüwer, N.; Sugnaux, C.; Tugulu, S.; Klok, H.-A. Polymer Brushes via Surface-Initiated Controlled Radical Polymerization: Synthesis, Characterization, Properties, and Applications. Chem. Rev. 2009, 109, 5437-5527.

(23) Zdyrko, B.; Luzinov, I. Polymer Brushes by the "Grafting to" Method. Macromol. Rapid Commun. 2011, 32 , 859-869.

(24) Minko, S. Grafting on Solid Surfaces: "Grafting to" and "Grafting from" Methods. Polymer Surfaces and Interfaces; Springer: Heidelberg, 2008; pp 215-234.

(25) Sweat, D. P.; Kim, M.; Yu, X.; Gopalan, P. A single-component Inimer Containing Cross-linkable Ultrathin Polymer Coating for Dense Polymer Brush Growth. Langmuir 2013, 29, 3805-3812.

(26) Zhang, F.; Shi, Z. L.; Chua, P. H.; Kang, E. T.; Neoh, K. G. Functionalization of Titanium Surfaces via Controlled Living Radical Polymerization: from Antibacterial Surface to Surface for Osteoblast Adhesion. Ind. Eng. Chem. Res. 2007, 46, 9077-9086.

(27) Friis, J. E.; Brøns, K.; Salmi, Z.; Shimizu, K.; Subbiahdoss, G.; Holm, A. H.; Santos, O.; Pedersen, S. U.; Meyer, R. L.; Daasbjerg, K.; Iruthayaraj, J. Hydrophilic Polymer Brush Layers on Stainless Steel Using Multilayered ATRP Initiator Layer. ACS Appl. Mater. Interfaces 2016, 8, 30616-30627.

(28) Barthele' my, B.; Devillers, S.; Minet, I.; Delhalle, J.; Mekhalif, Z.' Induction Heating for Surface Triggering Styrene Polymerization on Titanium Modified with ATRP Initiator. J. Colloid Interface Sci. 2011, 354, 873-879.

(29) Boissezon, R.; Muller, J.; Beaugeard, V.; Monge, S.; Robin, J.-J. Organophosphonates as Anchoring Agents onto Metal Oxide-based Materials: Synthesis and Applications. RSC Adv. 2014, 4, 35690-35707.

(30) Tintore, M.; Mazzini, S.; Polito, L.; Marelli, M.; Latorre, A.; Somoza, Á.; Aviño, A.; Fa' brega, C.; Eritja, R. Gold-coated Super-' paramagnetic Nanoparticles for Single Methyl Discrimination in DNA Aptamers. Int. J. Mol. Sci. 2015, 16, 27625-27639.

(31) Rundqvist, J.; Hoh, J. H.; Haviland, D. B. Poly (ethylene glycol) self-assembled monolayer island growth. Langmuir 2005, 21, 2981-2987.

(32) Walter, A.; Garofalo, A.; Parat, A.; Jouhannaud, J.; Pourroy, G.; Voirin, E.; Laurent, S.; Bonazza, P.; Taleb, J.; Billotey, C.; Vander Elst, L.; Muller, R. N.; Begin-Colin, S.; Felder-Flesch, D. Validation of a Dendron Concept to Tune Colloidal Stability, MRI Relaxivity and Bioelimination of Functional Nanoparticles. J. Mater. Chem. B 2015, 3, 1484-1494. 
(33) Guerrero, G.; Mutin, P. H.; Vioux, A. Anchoring of Phosphonate and Phosphinate Coupling Molecules on Titania Particles. Chem. Mater. 2001, 13, 4367-4373.

(34) Cao, E.; Prouzet, E.; Heroguez, V. Organic' -inorganic Hybrid Materials Designed by Controlled Radical Polymerization and Mediated using Commercial Dual Functional Organophosphorous Coupling Agents. New J. Chem. 2014, 38, 6081-6087.

(35) Zhang, K.; Li, J.-A.; Deng, K.; Liu, T.; Chen, J.-Y.; Huang, N. The Endothelialization and Hemocompatibility of the Functional Multilayer on Titanium Surface Constructed with type IV collagen and Heparin. Colloids Surf., B 2013, 108, 295-304.

(36) Vergnat, V.; Pourroy, G.; Masson, P. Enhancement of Styrene Conversion in Organic/inorganic Hybrid Materials by using Malononitrile in Controlled Radical Polymerization. Polym. Int. 2013, 62, 878-883.

(37) Nosaka, Y.; Nosaka, A. Y. Generation and Detection of Reactive Oxygen Species in Photocatalysis. Chem. Rev. 2017, 117, 11302-11336.

(38) Oi, L. E.; Choo, M.-Y.; Lee, H. V.; Ong, H. C.; Hamid, S. B. A.; Juan, J. C. Recent advances of titanium dioxide (TiO2) for green organic synthesis. RSC Adv. 2016, 6, 108741-108754.

(39) Luo, W.; Zafeiratos, S. Tuning Morphology and Redox Properties of Cobalt Particles Supported on Oxides by an in between Graphene Layer. J. Phys. Chem. C 2016, 120, 14130-14139.

(40) Sekler, J.; Steinmann, P. A.; Hintermann, H. E. The Scratch Test: Different Critical Load Determination Techniques. Surf. Coat. Technol. 1988, 36, 519-529.

(41) Kokubo, T.; Kushitani, H.; Sakka, S.; Kitsugi, T.; Yamamuro, T. Solutions Able to Reproduce in vivo Surfacestructure Changes in Bioactive Glass-ceramic A-W3. J. Biomed. Mater. Res. 1990, 24, 721-734.

(42) Tanahashi, M.; Yao, T.; Kokubo, T.; Minoda, M.; Miyamoto, T.; Nakamura, T.; Yamamuro, T. Apatite Coating on Organic Polymers by a Biomimetic Process. J. Am. Ceram. Soc. 1994, 77, 2805-2808.

(43) Xia, Y.; Boey, F.; Venkatraman, S. S. Surface Modification of Poly(l-lactic acid) with Biomolecules to Promote Endothelialization. Biointerphases 2010, 5, FA32-FA40.

(44) Zhang, K.; Li, J.-A.; Deng, K.; Liu, T.; Chen, J.-Y.; Huang, N. The Endothelialization and Hemocompatibility of the Functional Multilayer on Titanium Surface Constructed with Type IV Collagen and Heparin. Colloids Surf., B 2013, 108, 295-304.

(45) Vergnat, V.; Roland, T.; Pourroy, G.; Masson, P. Effect of Covalent Grafting on Mechanical Properties of TiO2/polystyrene Composites. Mater. Chem. Phys. 2014, 147, 261-267.

(46) Boyer, C.; Corrigan, N. A.; Jung, K.; Nguyen, D.; Nguyen, T.-K.; Adnan, N. N. M.; Oliver, S.; Shanmugam, S.; Yeow, J. Copper Mediated Living Radical Polymerization (Atom Transfer Radical Polymerization and Copper(0) Mediated Polymerization): From Fundamentals to Bioapplications. Chem. Rev. 2015, 116, $1803-1949$.

(47) Wang, J.-S.; Matyjaszewski, K. Controlled/“living” Radical Polymerization. Atom Transfer Radical Polymerization in the Presence of Transition-metal Complexes. J. Am. Chem. Soc. 1995, 117, 5614-5615.

(48) Tao, W.; Fei, F.; Yue-Chuan, W. Structure and Thermal Properties of Titanium Dioxide-polyacrylate Nanocomposites. Polym. Bull. 2006, 56, 413-426.

(49) Tanaka, S.-I.; Tobimatsu, H.; Maruyama, Y.; Tanaki, T.; Jerkiewicz, G. Preparation and Characterization of Microporous Layers on Titanium. ACS Appl. Mater. Interfaces 2009, 1, 2312-2319.

(50) Kim, H.-M.; Miyaji, F.; Kokubo, T.; Nakamura, T. Preparation of Bioactive Ti and Its Alloys via Simple Chemical Surface Treatment. J. Biomed. Mater. Res. 1996, 32, 409-417.

(51) Mohammed, H. I.; Abdel-Fattah, W. I.; Sallam, M. A.; El-Sayed, M. E.; Talaat, M. S. E. H.; Faerber, J.; Pourroy, G.; Roland, T.; Carrado, A. Calcium Phosphate Coating on Ti 6Al4V by Autocatalytic route. Bioinspired, Biomimetic Nanobiomater. 2012, 1, 221-228. 
(52) Carrado, A.; Perrin-Schmitt, F.; Le, Q. V.; Giraudel, M.; Fischer, C.; Koenig, G.; Jacomine, L.; Behr, L.; Chalom, A.; Fiette, L.; Morlet, A.; Pourroy, G. Nanoporous Hydroxyapatite/sodium Titanate Bilayer on Titanium Implants for Improved Osteointegration. Dent. Mater. 2017, 33, 321-332.

(53) Jiaming, Z.; Rui, L.; Jianying, H.; Jiayan, C.; Xurong, L.; Yutai, L.; Yousi, Z. A Novel Rate-accelerating Additive for Atom Transfer Radical Polymerization of Styrene. J. Polym. Sci., Part A: Polym. Chem. 2007, 45, 4082-4090.

(54) Ajami, E.; Aguey-Zinsou, K.-F. Functionalization of Electropolished Titanium Surfaces with Silane-based Self-assembled Monolayers and Their Application in Drug Delivery. J. Colloid Interface Sci. 2012, 385, $258-267$.

(55) Wang, Q.; Zhong, L.; Sun, J.; Shen, J. A facile Layer-by-Layer Adsorption and Reaction Method to the Preparation of Titanium Phosphate Ultrathin Films. Chem. Mater. 2005, 17, 3563-3569.

(56) Duan, G.; Zhang, C.; Li, A.; Yang, X.; Lu, L.; Wang, X. Preparation and Characterization of Mesoporous Zirconia Made by using a Poly(methyl methacrylate) Template. Nanoscale Res. Lett. 2008, 3, 118-122.

(57) Minet, I.; Delhalle, J.; Hevesi, L.; Mekhalif, Z. Surface-initiated ATRP of PMMA, PS and diblock PS-bPMMA Copolymers from Stainless Steel Modified by 11-(2-bromoisobutyrate)-undecyl-1-Phosphonic Acid. J. Colloid Interface Sci. 2009, 332, 317-326.

(58) Reggente, M.; Natali, M.; Passeri, D.; Lucci, M.; Davoli, I.; Pourroy, G.; Masson, P.; Palkowski, H.; Hangen, U.; Carrado, A.; Rossi, M. Multiscale Mechanical Characterization of Hybrid Ti/

PMMA Layered Materials. Colloids Surf., A 2017, 532, 244-251. (59) Kuiry, S. Advanced Scratch Testing for Evaluation of Coatings. Tribology and Mechanical Testing; Bruker Nano Surfaces Division: USA, 2012.

(60) Johnson, I.; Liu, H. A Study on Factors Affecting the Degradation of Magnesium and a Magnesium-yttrium Alloy for Biomedical Applications. PLoS One 2013, 8, No. e65603. 